\title{
Robotic Path Planning using Genetic Algorithm in Dynamic Environment
}

\author{
Toolika Arora \\ ITM University, Gurgaon, India
}

\author{
Yogita Gigras \\ ITM University, Gurgaon, India
}

\author{
Vijay Arora \\ Dronacharya College of \\ Engineering, Gurgaon, India
}

\begin{abstract}
Path planning is one of the important part of robotics. In this paper a path planning method based on genetic algorithm is proposed for finding path for mobile robot in dynamic environment. Here the genetic algorithm is applied at a point in the problem space not at the complete space. In this study the performance of the algorithm in terms of execution time and path length is evaluated using MATLAB environment.
\end{abstract}

\section{Keywords}

Genetic Algorithm, Mobile Robot, Path Planning.

\section{INTRODUCTION}

While studying Robotics Path planning is considered to be a very important topic. The mobile robot has to move from start position to the end position while avoiding obstacles in a environment containing obstacles. The path should be optimal based on some criteria like path length and execution time. Till now a lot of researchers from all over the world have done many studies on robotic path planning using various algorithms which include Meta heuristic algorithms like Neural Network, Ant Colony Optimization (ACO), Particle Swarm Optimization (PSO), Genetic Algorithm (GA)and so on. Path planning can be done in online mode and offline mode. In offline mode the robot has the roadmap of the environment. It is known by the robot where the obstacles are placed and the environment is static i.e obstacles are fixed to position. On the other hand in online mode the information about the space is obtained at the time of motion of robot. This information include present position of robot and local information. Here the environment is dynamic. Considering the work proposed by previous researchers. In [5] [7] improved simulated annealing combined with artificial neural network for finding a solution for path planning problem. In [6] ACO is applied in dynamic environment using grid environment. Genetic algorithm has been utilized by various researchers differently in their work. In [1] Adaptive Genetic Algorithm and in [2] Multiple Objective Optimization Genetic Algorithm are proposed for path planning in static environment. In [3] improved genetic algorithm is proposed for dynamic environment. A comparison is made between two techniques ACO and Genetic Algorithm in [4]. Both are applied to same dynamic environment and their performances are measured and compared. Till now most of the research papers studied generally contain grid based problem space in which the space is divided into squares of equal dimensions. In this paper the environment used for robot is 2-D space. In
[8] also ACO and PSO are used in 2-D space in dynamic environment.

\section{GENETIC ALGORITHM}

It is a very popular meta-heuristic technique for solving optimization problems. In this a population of candidate solutions is evolved to search better solution. It is based on natural selection. Where an initial set of population which are the possible solution to the problem are generated randomly and then the fitness value of each solution is calculated by applying the objective function of the problem. Then the more fit solutions are selected and genetic operations mutation and crossover are applied to them to generate a new population of more fit candidates. This thing repeats till the maximum number of iterations are reached or satisfactory fitness value is attained. This technique is utilized successfully by various researchers for solving various optimization problems like travelling salesman problem, job scheduling, path planning etc. The results obtained are also analysed. In this paper genetic algorithm is used for robotic path planning.

\section{PROBLEM DESCRIPTION}

Suppose we have a moving space for robot of 200X200. The left bottom corner $\left(x_{1}, y_{1}\right)$ is the starting point while the right top corner $\left(x_{2}, y_{2}\right)$ is destination point of the path. Inside the moving space there are 20 randomly generated rectangular obstacles therefore the position of obstacles is not known to the robot (Dynamic System). The size of obstacles is also variable. The goal is to determine a shortest path from source to destination by avoiding obstacles in the optimal time using genetic algorithm. In this configuration source point is represented by red rectangle and destination point by blue diamond.

\section{PROPOSED SOLUTION}

Initially the robot started from source and following the diagonal directional path from source towards the destination which is the minimum path. Direction is calculated as:

$$
\theta=\tan \frac{x 1}{y 1}
$$

And coordinates are calculated as:

$$
\begin{aligned}
& x 1=x 1+\cos \theta \\
& y 1=y 1+\sin \theta
\end{aligned}
$$


While moving on the path if an obstacle arise in between then robot takes 3 steps back and reach at position (x,y) and then use genetic algorithm to determine the next path.

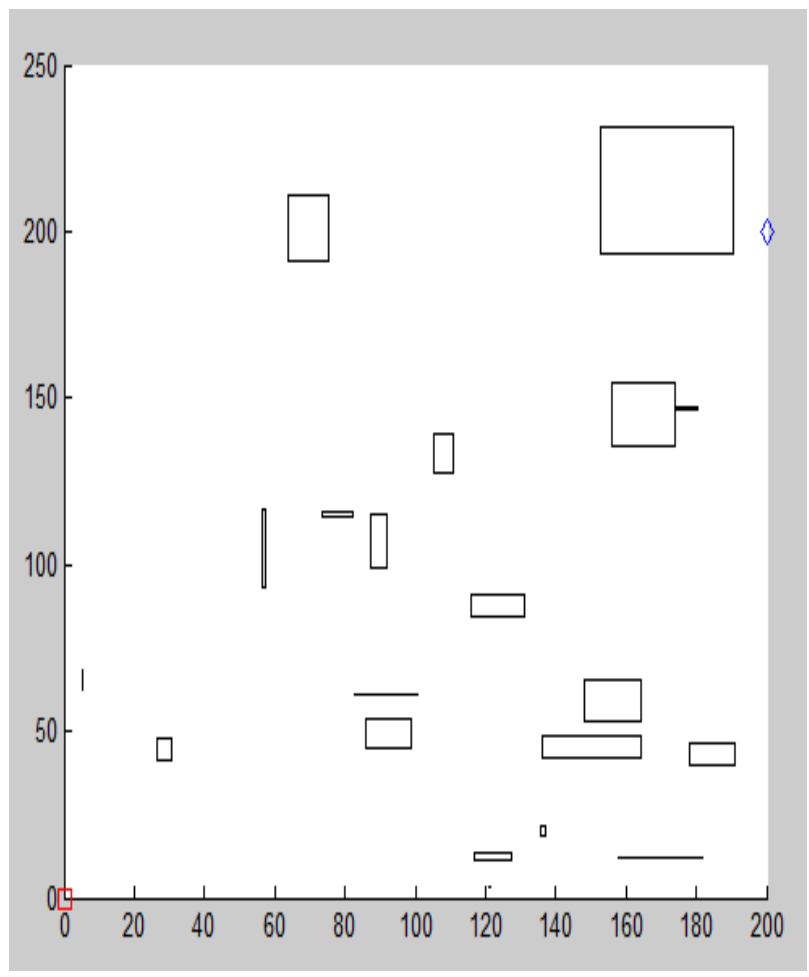

Figure 1 problem configuration.

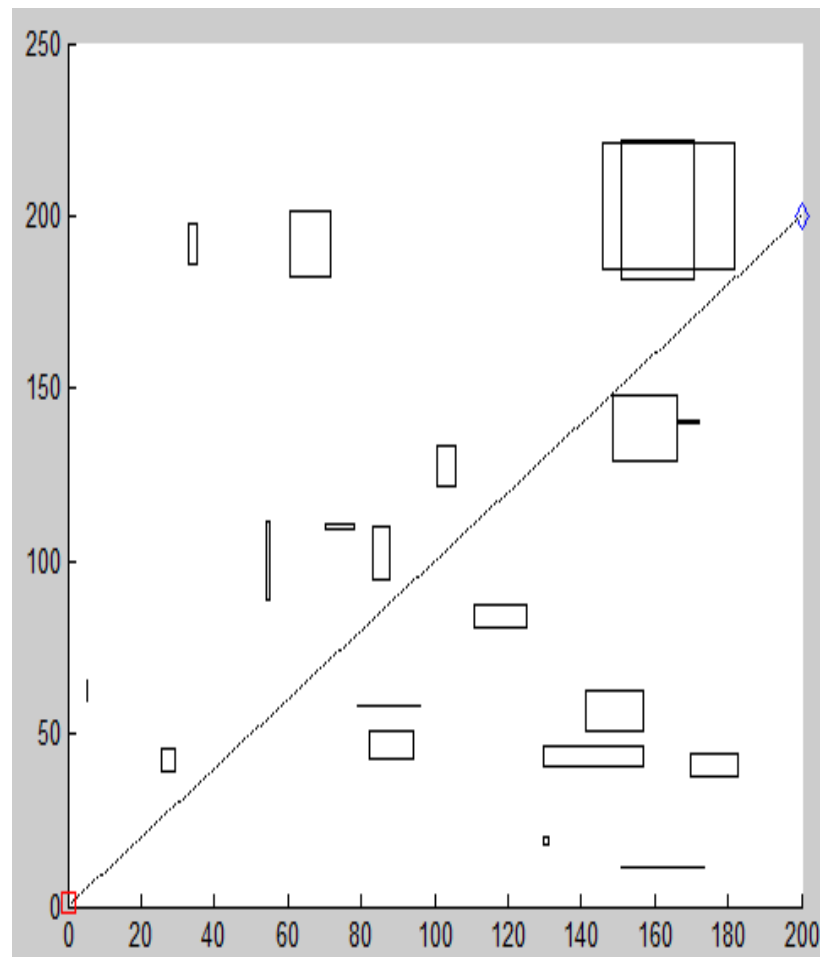

Figure 2 Goal configuration

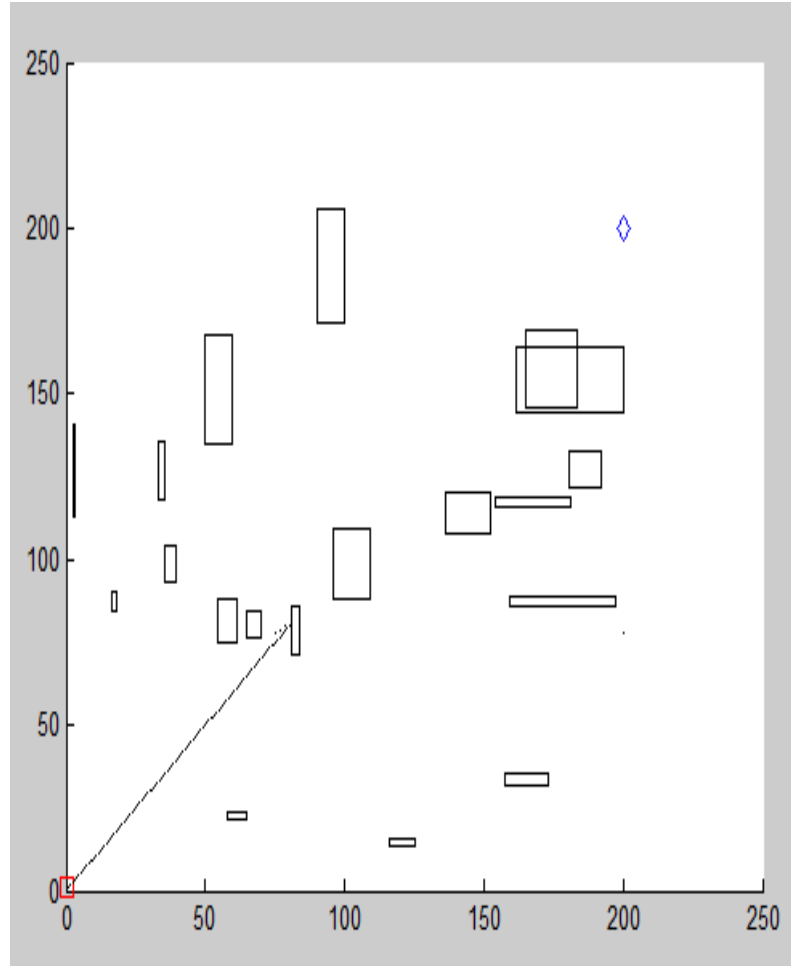

Figure 3 After colliding with obstacle. 


\section{FLOW DIAGRAM}

Initially start with starting point and follow directional path untill end point is reached or any obstacle is met.

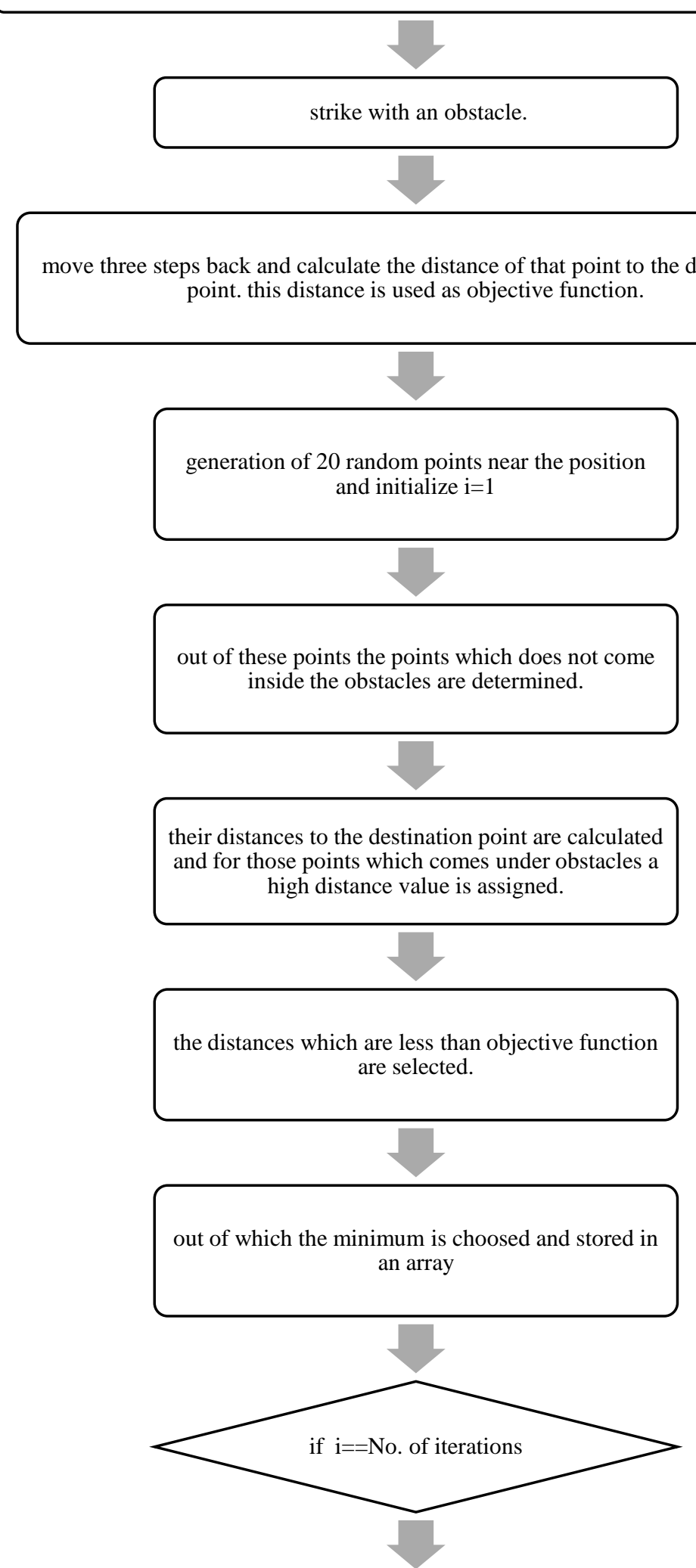

the minimum out of the array is determined and selected as the solution. the directional path is followed and above steps are repeat if any other obstacle encountered. 


\section{FITNESS/OBJECTIVE FUNCTION}

It is the distance between the position where the robot stop and the destination.

$$
\text { minDistance }=\sqrt{\left(x-x_{2}\right)^{2}+\left(y-y_{2}\right)^{2}}
$$

A solution that is to be selected among candidate solutions should be less than or equal to this distance.

\subsection{Natural Selection}

Out of 20 random points feasible points are determined which does not come inside any obstacle. Then these points are compared with objective function and the points having less or equal value to the objective value are selected as the fittest point.

\subsection{Mutation and Crossover}

Mutation and crossover are applied on the points which are selected by natural selection process.as follows:

For Mutation a parameter pm is set to a value. For every chromosome in the population a random number (rand1) is generated if it is greater than the pm than mutation is applied to it as follows:

$$
\begin{aligned}
& x(i)=\operatorname{rand} 1+x(i) \\
& y(i)=\operatorname{rand} 1+y(i)
\end{aligned}
$$

For Crossover another parameter pc is set to a value. Here again a random number is generated for every chromosome if it is greater than pc than crossover is applied as:

$$
\begin{aligned}
& x(i)=\operatorname{rand} 2+x(i-1) \\
& y(i-1)=\operatorname{rand} 2+y(i)
\end{aligned}
$$

\subsection{Algorithm}

1.) Initial Population of 20 random points $\left(x_{i}, y_{i}\right) i=$ 1 to 20 is generated near the current position.

2.) Out of these 20 points those points are determined that comes inside any obstacle. Their distance to the destination are set to a very large value. So that those points are not feasible for selection.

3.) Then distances of feasible points to destination is calculated.(fitness value)

$$
\operatorname{distance}(i)=\sqrt{\left(x_{i}-x_{2}\right)^{2}+\left(y_{i}-y_{2}\right)^{2}}
$$

4.) These distances are the candidate solutions.

5.) Then these candidate solutions are compared to the objective function value and to each other.

6.) The candidate solution which has minimum value is selected as good solution.

7.) This solution is saved somewhere and then Crossover and mutation are applied and a new population is created.

8.) Steps 2 to 7 are repeated $\mathrm{N}$ times (number of iterations).

9.) The solution having the minimum value among the saved solutions is taken as the Final solution. Robot proceed toward the destination point using this solution and if any other obstacle arise in between then this process is repeated again.

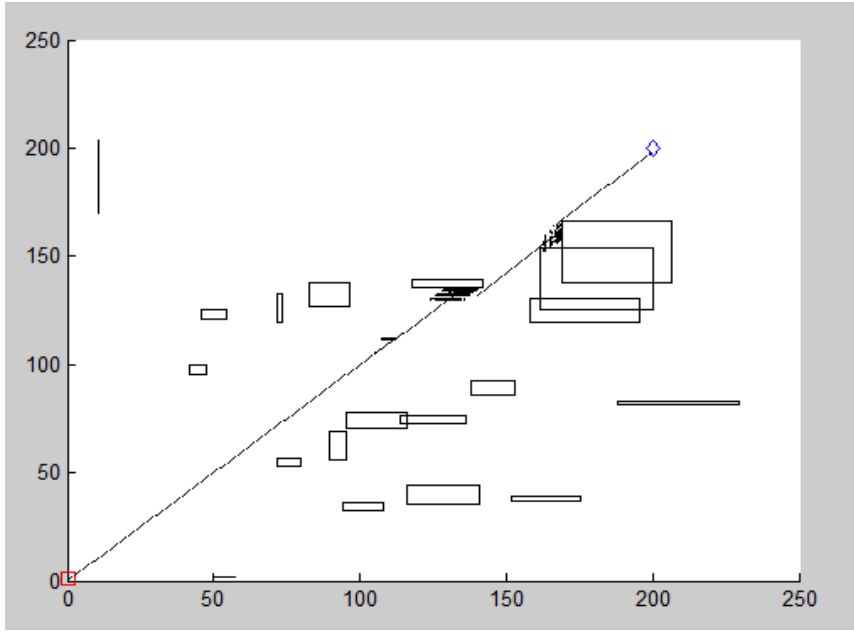

Figure 4 Solution obtained after applying GA.

\section{RESULT}

On implementing the above GA algorithm in Matlab CPU Time in seconds for different number of iterations is calculated.

Table1 Number of iterations Vs CPU Time (sec).

\begin{tabular}{|l|l|}
\hline No. of iterations & GA \\
\hline 10 & 26 \\
\hline 20 & 102 \\
\hline 30 & 122 \\
\hline 40 & 139 \\
\hline
\end{tabular}

\section{CONCLUSION}

In this paper a Dynamic Environment is used and Genetic Algorithm is performed successfully on it. The above simulation results obtained are high efficient than results obtained by other algorithms used for robotic path planning. Genetic algorithm is very effective and efficient algorithm. Advantages of Genetic algorithm can be applied to variety of applications. It is expected to generate next paper on moving obstacles by using any meta-heuristic technique.

\section{REFERENCES}

[1] Chang ling Liu, Huai wang Liu, Jingyu Yang. "A path planning method based on adaptive genetic algorithm for mobile robot." Journal of information \& computational science 8:5 (2011) 808-814.

[2] O. Castillo \& L. Trujillo. "Multiple objective optimization genetic algorithms for path planning in autonomous mobile robots." International Journal of computers, systems \& signals, Vol. 6, No. 1, 2005.

[3] Xuan Zou, Bin Ge, Peng Sun.'Improved genetic algorithm for dynamic path planning." IJICS Volume 1, Issue 2, May 2012 PP 16-20.

[4] Fatmeh Khosravi purain, Fardad Farokhi, Reza Sabbaghi Nadoosham"Comparing the performance of genetic algorithm \& Ant colony optimization algorithm for mobile robot path planning in dynamic environment with different complexities." Journal of Academic \& Applied Studies Vol. 3(2) February 2013, pp 29-44. 
[5] Meijuan Gao, Jing wen Tian. "Path planning for mobile robot based on Improved Simulated Annealing Artificial Neural Network." Third International Conference on Natural Computation (ICNC 2007) IEEE 0-7695-2875-9/07.

[6] Micheal Brand, Micheal Masuda, Nicole Wehner, Xiao Hua Yu. "Ant colony optimization algorithm for robot path planning." 2010 International conference on computer design and applications (ICCDA 2010)

[7] Xianmin Wei. "Robot path planning based on Simulated Annealing \& Artificial Neural networks. " Research Journal of Applied Sciences, Engineering \& Technology 6(1): 149-155, 2013.
[8] Yogita Gigras, Kusum Gupta. "Meta-heuristic algorithm for robotic path planning."IJCA(09758887) Volume-85, No. 3 January 2014.

[9] Alpa Reshamwala. "Robot Path Planning using An Ant Colony Optimization Approach: A survey." IJARAI Vol. 2, No. 3, 2013.

[10] Er. Waghoo Parvez, Er. Sonel Dhar. " Path planning optimization using Genetic Algorithm : A Literature Review." International Journal of computational Engineering Research $\|$ Vol. 03\|Issue, 4\|.

[11] Aditia Hermanu, Theadore W. Manikas, Kaveh Ashenayi, Roger L. Wain Wright. "Autonomous robot navigation using a genetic algorithm with an efficient genotype structure 